\title{
Tunable and Memorable Optical Devices with One-Dimensional Photonic-Crystal/Liquid-Crystal Hybrid Structures
}

Po-Chang Wu and Wei Lee

Additional information is available at the end of the chapter

http://dx.doi.org/10.5772/48127

\section{Introduction}

\subsection{Concept of defect-mode tunability in a one-dimensional photonic- crystal/liquid-crystal hybrid cell}

Photonic crystals (PCs) are a special class of artificial structures with spatially periodic dielectric permittivity and their investigations stem from 1987 when both Yablonovitch and John independently demonstrated their own findings (Yablonovitch, 1987; John, 1987). The most attractive feature of PCs is the existence of photonic bandgap (PBG), characterized by the spatial distribution of refractive index or dielectric constant. The PBG of PCs is analogous to the electronic bandgap of semiconductors, meaning that certain photons will be localized and forbidden in propagation through PCs. According to this characteristic, PCs can be of wide use; thus, various types of PCs have successively been proposed and devised for photonic applications. (Fleming \& Lin, 1999; Imada et al., 1999; Knight, 2003; Krauss et al., 2000; Nelson et al., 2000; Park, 1999). If a defect layer is infiltrated in a PC to disrupt its periodicity, partial defect modes that allow the transmission of photons at specific wavelengths will be generated within the PBG. Based on this design of PCs with defect layers, several photonic devices made of two- or three-dimensional PCs have been suggested for lasers (Painter et al., 1999), optical fibers (Knight et al., 1998), and some other applications (Blanco et al., 2000; Chow et al., 2000). Notably, the spectral profile of a PC can be more flexible when the PBG is intrinsically tunable or when the PC is composed of a tunable defect layer.

Liquid crystals (LCs) are anisotropic materials whose physical properties such as electrical and optical anisotropy can be tuned by the electric field, magnetic field, temperature, and the like in that the LC molecules are susceptible to external stimuli. Depending on the 
molecular orientation, LCs can serve as a phase retardation medium and an optical polarization rotator to manipulate the incoming light via the electrically controlled birefringence and polarization rotation, respectively. As a result, LCs are widely applied to various types of currently used electro-optical devices, especially the information display. As a matter of fact, certain LCs such as the cholesteric LC (CLC), blue phase LC (BP LC) and ferroelectric LC (FLC) can be regarded as PCs due to their periodical orientation of molecular helix. Among them, the PBG of CLCs depends on the LC and chiral parameters. The tunable CLC PCs can thus be realized by adjusting the external factors such as temperature (Morris et al., 2005; Hung et al., 2000), light irradiation (Bobrovsky et al., 2003; Lin et al., 2005), or electric field (Choi et al., 2009; Lin et al., 2005; Yu et al., 2005). Some other tunable PCs based on FLC (Kasano et al., 2003) or BP LC (Yokoyama et al., 2006) have also been demonstrated.

By inserting a LC as defect layer in the PC, tunabilities in the profiles of defect modes are expected. The first tunable PC/LC hybrid structure was developed by Ozaki et al. when they used a planar aligned nematic LC as a defect layer sandwiched between two onedimensional (1D) periodical multilayers (i.e., dielectric mirrors) and successfully demonstrated the electrically tunable wavelength of defect modes (Ozaki et al., 2002). Their concept of tunability in the 1D PC/LC cell can briefly be described as follows: Figure 1 illustrates the setup for investigation of the transmission spectra of the PC/LC cell. Note that the configuration includes a single linear polarizer in front of the PC/LC cell. The LC with positive dielectric anisotropy is aligned homogeneously along the $x$-axis whereas the incoming light propagates in the $z$-axis. The multilayer consists of two dielectric materials, with high- and low-refractive-index layers stacked alternatively. When the incoming light is normally incident to the PC/LC cell with its polarization direction parallel to the LC molecular axis, the optical path length (OPL) is contributed by the sole extraordinary refractive index $\left(n_{\mathrm{e}}\right)$. The appearance of peaks in the PBG of the transmission spectrum thus represents extraordinary defect modes. Once an electric field is applied across the cell to align the molecule along the $z$-axis, the contribution of the ordinary refractive index $\left(n_{\mathrm{o}}\right)$ to the OPL leads to the shift of defect modes to the shorter wavelength in the spectrum. On the contrary, the wavelength positions of all defect modes remain unchanged under the field-on and field-off conditions when the polarization direction of the impinging light becomes parallel to the $y$-axis due to the equal contribution of the OPL. It is concluded that the tunability of defect modes is attributed to the change in refractive index as well as the OPL manipulated by the electric field. After the publication of the milestone paper by Ozaki et al. (Ozaki et al., 2002), the idea has been extended to the 1D PC/CLC color-tunable lasing (Matsuhisa et al., 2006, 2007; Ozaki et al., 2003a, 2005; Park et al., 2009) and fast-response PC/LC structure (Ozaki et al., 2003b). Furthermore, based on this mechanism, several approaches, such as varying the incident angle of light (Arkhipkin et al., 2007) and temperature (Arkhipkin et al., 2008) or using magnetic field (Zyryanov et al., 2008) to adjust the optical anisotropy of LCs, have successively been proposed for realization of tunable 1D PC/LC cells. Particularly, Zyryanov and colleagues further took the investigation of a 1D PC/LC cell to a new stage by setting the hybrid cell between crossed polarizers (Zyryanov et 
al., 2010). Their results conclude some attractive features for the tunable mechanism of defect modes within the PBG. First, the shift of defect-mode wavelengths depends on the change in effective refractive index (neff) (Zyryanov et al., 2008, 2010). The decrease in $n_{\text {eff }}$ gives rise to the blueshift of defect-mode wavelengths whereas redshift occurs as $n_{\text {eff }}$ increases. In addition, a larger number of defect modes can be obtained by using LC with high refractive index or by increasing the thickness of the defect layer, enabling the 1D PC/LC structure to be applicable for the use of a narrow band filter. Moreover, they experimentally and theoretically demonstrated that the transmission tunability of defect modes can be achieved by placing the cell between crossed polarizers to allow "interference" between two orthogonal polarization components through vector sum in the projection direction along the transmission axis of the analyzer. As a result, the transmittance is increased when the defect-mode wavelength of an extraordinary component overlaps that of an ordinary one (Zyryanov et al., 2008).

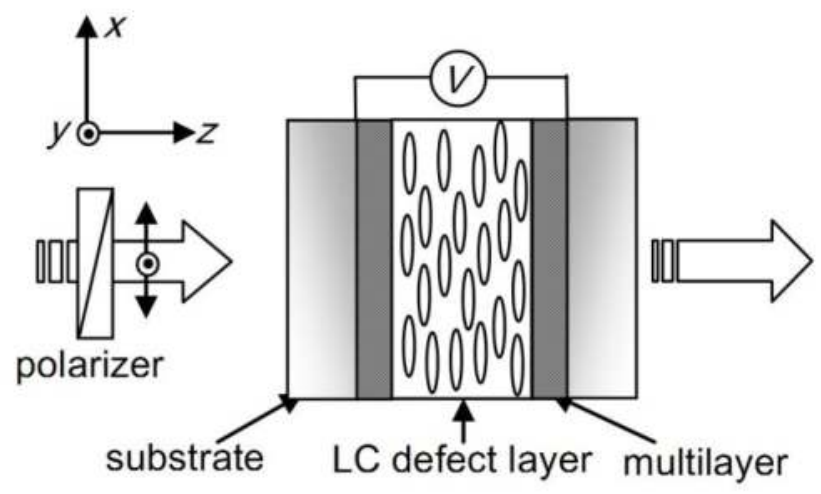

Figure 1. Schematic of a 1D PC/LC hybrid structure in a typical experimental setup.

\subsection{Overview of the development of one-dimensional photonic crystals with a dynamic-mode liquid crystal}

Current available LC devices can be classified into two categories, dynamic-mode (DM) and memory-mode (MM)LCs, according to their operation as a function of applied electric field. The LC molecules in the DMLC have only one stable state, determined by the condition of the alignment film, and they are continuously reoriented with applied voltage. On the contrary, there are two or multistable states in the MMLC and the stable states can be switched from one to another by the applied voltage. In the DMLC, it can serve as a phase retarder or optical rotator and the light passing them is modified by electrically controlled birefringence (ECB) and polarization rotation effect, respectively. For the 1D PC/LC cells mentioned in Section 1.1, the LC defect layers used are DMLCs acting as phase retarders. The mechanism of the tunable defect modes in these cells can thus be explained in terms of the ordinary and extraordinary refractive indices (Ozaki et al., 2002, 2004b; Zyryanov et al., 2008). On the other hand, the twisted-nematic (TN) LC, in which the molecular orientation 
exhibits $90^{\circ}$ twist, acts as an optical polarization rotator so that the light passing through the TN LC is characterized by the rotation of polarization. The hybrid structure configured by a 1D PC and a TN LC was demonstrated in 2010 (Lin et al., 2010). Several phenomena attributable to the adiabatic following are quite different from the tunability mechanism mentioned in the preceding section. To realize how the adiabatic following enables optical tunability, described here are the spectral characteristics of an electrically controlled 1D $\mathrm{PC} / \mathrm{TN}$ cell acquired in both the single-polarizer (SP) and crossed-polarizer (CP) schemes.

In the study reported by Lin and coworkers (Lin et al., 2010), the $90^{\circ} \mathrm{TN}$ LC modes are divided into three groups, depending on the polarization angle $\beta$ between the transmission axis of the first polarizer (i.e., input polarization) and the director axis (i.e., averaged molecular axis) lying in the front substrate. They are the ordinary-mode (O-mode), extraordinary-mode (E-mode), and mixed-mode (M-mode) TN satisfying the conditions of $\beta$ $=90^{\circ}, 0^{\circ}$, and $45^{\circ}$, respectively. It is worth mentioning that the M-mode TN considered by Lin et al. is only a specific one. Generally, the M-mode TN, abbreviated as MTN, combines the polarization-rotation effect as well as birefringence effect. A traditional TN is primarily characterized by its polarization-rotation effect, which is manifested when the multiplication of the mesogenic bulk thickness and the optical birefringence (i.e., optical anisotropy), $d \Delta n$, is larger than the Gooch-Tarry first minimum condition, namely, $(\sqrt{3} / 2) \lambda \approx 0.866 \lambda$, where $\lambda$ is the wavelength (Gooch \& Tarry, 1975). If $d \Delta n$ is smaller than that condition, then the polarization-rotation effect is incomplete. Another condition of MTN is that $0^{\circ}<\beta<90^{\circ}$ without the requirement of the twisted angle to be $90^{\circ}$. Under such a circumstance, birefringence effect will also take place. The triumph of MTN is its wide use in reflective LC displays, including pico projectors ( $\mathrm{Wu} \& \mathrm{Wu}, 1996)$. Figure 2 shows the phenomenon of the wavelength shift of defect modes in two PC/TN cells impregnated with two different nematic LC materials. Note that the birefringence of the LC material E7 is higher than that of CYLC43. Unlike the ECB-based tunable defect modes in some specific PC/LC cells, in which the defect modes for the ordinary-ray are independent of the applied voltage, the defectmode wavelengths in both the E-mode and O-mode PC/TN cell show blueshift when the applied voltage increases. Compared with the O-mode configuration, the E-mode has a more perceptible shift in wavelength (blueshift) due to significant change (decrease) in effective refractive index, dedicated by the unwinding process of the molecular helix. It is also clear from Fig. 2 that the extent of buleshift for E7 is greater than that for CYLC43. This result can be explained by their Mauguin parameters (Gooch \& Tarry, 1975)

$$
\mathrm{u}=\frac{2 \mathrm{~d} \Delta \mathrm{n}}{\lambda},
$$

giving superior adiabatic following capability of E7 (Lin et al., 2010). A perfect adiabatic following in the TN LC cell would enable the linearly polarized light to traverse the LC layer with the rotation of the molecular twist, which makes $n_{\mathrm{eff}}$ for the incident beam nearly equal to $n_{\mathrm{e}}$ in E-mode and $n_{\mathrm{o}}$ in O-mode. In fact, the elliptic polarization can hardly be avoided in the TN LC (Yeh \& Gu, 1999a); thus, neff is no longer a constant in the O-mode TN LC but becomes a weak function of applied voltage. As a result, small shifts for the defect modes are observed in the O-mode PC/TN cells. 


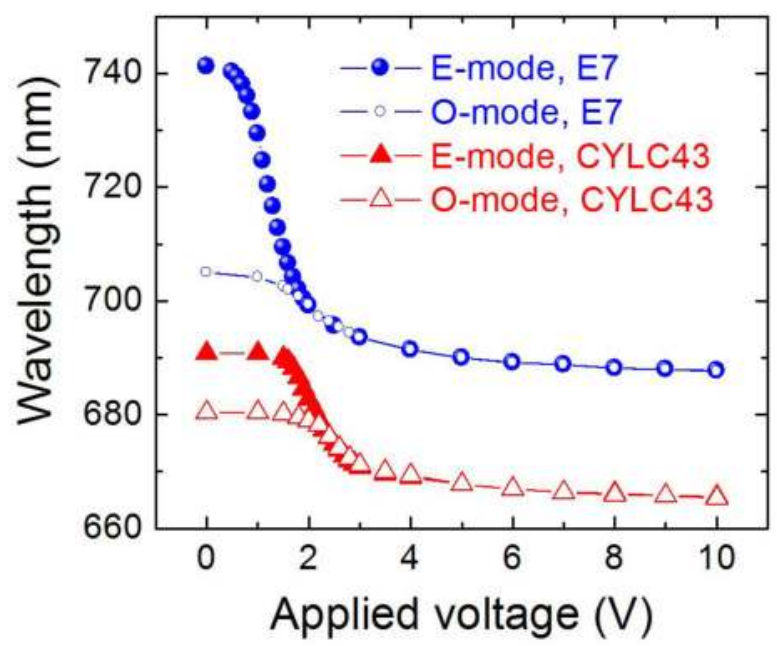

Figure 2. Voltage-induced blueshift of defect modes for two comparative 1D PC/LC cells with distinct defect materials (adapted from Lin et al., 2010).

Figure 3 demonstrates an integrated effect on the transmission of defect modes in the Mmode PC/TN cell. One can obviously identify that the peaks of the M-mode spectrum are located at the exactly same positions of the E-mode and O-mode peaks in wavelength. This result indicates that each defect mode in the M-mode PC/TN is contributed by both the adiabatic following and birefringence effects. Moreover, the intensity of the transmitted light in either E- or O-mode spreads to the other, making the integrated intensity of the peaks in the M-mode almost the same as that in either E- or M-mode. This implies that the M-mode spectrum is a superposition of those of both E-mode and O-mode. Interestingly, while looking carefully into the spectra of E- and O-mode, small satellite peaks accompanying the defect modes are observed, presumably due to the unavoidable elliptic polarization of light in a conventional TN cell. This phenomenon has left untouched in other types of 1D PC/LC cells. Recently, it has been explained by the Mauguin condition violation and the coupling between the slow semi-longitudinal mode (i.e., twisted extraordinary mode) and fast semi-transverse mode (i.e., twisted ordinary mode) PC/TN cell (Timofeev et al., 2012).

In regard to a typical $90^{\circ} \mathrm{TN}$ cell, the linearly polarized light rotated approximately by $90^{\circ}$ by the cell can almost "completely" pass the rear polarizer; i.e., the analyzer. There is no doubt that the spectra of the E-mode as well as the O-mode in the CP scheme (configured by two linear polarizers whose transmission axes are orthogonal to each other) are nearly identical to their corresponding ones under the SP scheme. Figure 4 reveals a distinctive profile in the spectrum for the M-mode because the light passing through the cell becomes non-linearly polarized light. Particularly, an outstanding peak, whose intensity is the strongest among the vast of peaks within the PBG, is located near $700 \mathrm{~nm}$ as shown in Fig. 4. This unique feature enables to extend the use of the PC/TN cell in the application of a 
monochromatic selector. In accordance with the simulation results published in the literature, this remarkable defect-mode peak is attributable to the intrinsic transmission characteristic of a MTN cell (Lin et al., 2010).

\subsection{Aim of this chapter}

Recently, electro-optical devices in line with the idea of energy saving and/or low power consumption become a popular research topic in that the green concept is globally promoted due to the great concern for energy shortage nowadays. The most representative one for alternative energy is the solar cell which has the ability to transfer natural energy from the sun to electric power. In view of the recent development in 1D PC/LC cells, the demonstrated features such as wavelength tunability and transmission tunablity enable their use for the application in various electro-optical devices, as described in Section 1.2. However, aforementioned features in 1D PC/LC cells are realized by the continuous-varying of electric field due to the use of DMLCs as the defect layer so that applications in green products are very limited. Lately, a new design of 1D PC with MMLC as a defect layer that brings the notion of multistability in defect modes has been demonstrated (Hsiao et al., 2011a, 2011c; Wu et al., 2011). In the 1D PC/MMLC cell, the spectral properties of defect modes in a memory state persist without applied voltage; such a cell supports a pathway for designing photonic devices with green concept. To interpret how the 1D PC/MMLC operates, this chapter reviews some previous works primarily done by this group and builds up the following three sections in the main body of this article to explicitly clarify the characteristics of such 1D PC/MMLC cells.

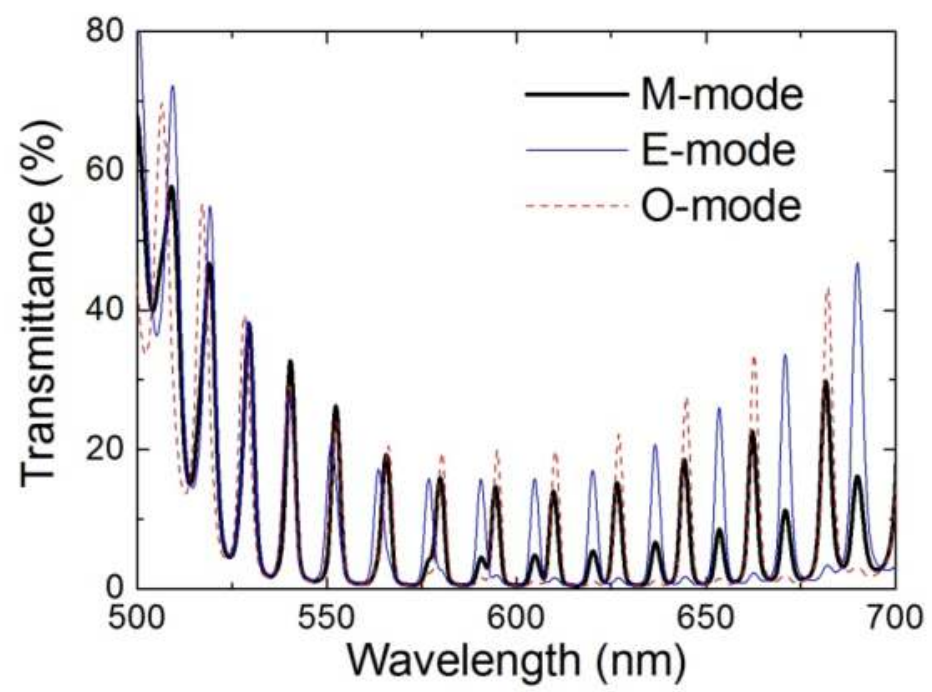

Figure 3. Transmission spectra of a PC/TN cell in the photonic bandgap in three different modes. The M-mode, E-mode and O-mode are represented by the thick solid line, thin solid line and dashed line, respectively (adapted from Lin et al., 2010). 


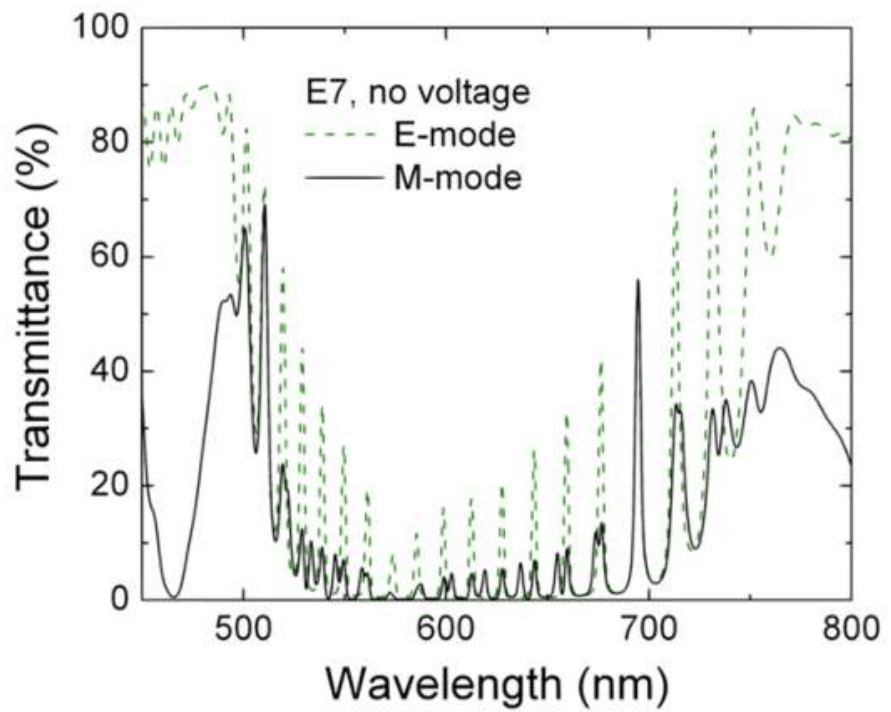

Figure 4. Transmission spectra of a $1 \mathrm{D}$ PC/TN cell at null voltage under crossed polarizers (adapted from Lin et al., 2010).

Among the recent development of MMLCs, the bistable or multistable cells using dualfrequency LCs (DFLCs) enable the switching between optically stable states by applying frequency-modulated voltage pulses. The DFLC is a kind of LC material whose sign of dielectric anisotropy $(\Delta \varepsilon)$ can be varied by the frequency of an externally applied electric field (Xianyu et al., 2009). The DFLC has a certain crossover frequency $\left(f_{c}\right)$ to discriminate the behavior of $\Delta \varepsilon$. While the frequency is lower than $f_{c}$, the $\Delta \varepsilon$ value is positive. Or it becomes negative if the frequency is higher than $f_{c}$. Based on this mechanism, various types of MMLC cells composed of DFLC are demonstrated in the literature (Hsiao et al., 2011b; Hsu et al., 2004; Jhun et al., 2006; Yao et al., 2009). Among them, the bistable chiralhomeotropic nematic (BHN) LC and dual-frequency cholesteric LC (DFCLC) have been used as defect layer in the 1D PC/MMLC cell (Hsiao et al., 2011a, 2011c; Wu et al., 2011). As such, Section 2 details the cell configuration and operation principles of these two MMLC modes so that one can grasp the switching mechanisms in the description in the next two sections. The configuration of a 1D PC/MMLC cell, including the design of multilayers is schematically depicted in Session 3. In addition, the optical properties and the tunability in the defect modes of the cell switching among the stable and voltage-sustained states are reported in Session 3 as well. To realize a low-power-consumption optical device, in Session 4 , the characteristics of defect modes in various memory states of both $\mathrm{PC} / \mathrm{BHN}$ and PC/DFCLC cells are further discussed. The features of defect-mode switching between two stable states are confirmed. Particularly, a new scheme of a tristable PC device based on DFCLC is demonstrated using a polymer-stabilized cholesteric texture (PSCT) as a defect layer (Hsiao et al., 2011). Finally, the key findings of the properties of the 1D PC/MMLC cells discussed in the preceding sections are summarized. In accordance with the concluding remarks, suitable device applications of the tunable 1D PC/MMLC hybrid structures are 
suggested in the last section of the chapter. A brief note will also be included on future perspectives of the development of 1D PC/MMLC cells.

\section{Operation principles of memory-mode liquid crystals}

\subsection{Bistable chiral-tilted homeotropic nematic liquid crystals}

The BHN LC possesses two optically stable states-tilted-homeotropic $(\mathrm{tH})$ and tilted-twist (tT) states, either of which remains optically stable without the need of continuous application of a voltage (Hsu et al., 2004). By means of a DFLC, switching between these two states is accomplished by the flow effect of LC molecules and frequency-revertible dielectric anisotropy of the DFLC. To fabricate a BHN LC cell, the thickness-to-pitch ratio $d / p$ and the pretilt angle $\theta$ of LC molecules, measured from the substrate plane, play crucial roles in the stability of the $\mathrm{tH}$ and $\mathrm{tT}$ states. Typically, the BHN LC cell is made in the high-pretilt-angle regime with $d / p$ around unity. To understand the optimized condition of $d / p$ and $\theta$, Liang and Lin determined the $d / p$ ranges of the BHN LC cells with various pretilt angles based on their experimental and simulation results (Liang \& Lin, 2007). They found that the $d / p$ range for obtaining stable $\mathrm{tH}$ and $\mathrm{tT}$ state in the BHN LC cell is 0.6-1.14, 0.68-1.21, and 0.82-1.24 when the pretilt angle is equal to $62^{\circ}, 72^{\circ}$, and $80^{\circ}$, respectively. In additional to the conventional $(0,2 \pi)$ BHN LC, the two stable states can also be found in the $(-\pi / 2,-3 \pi / 2)$ BHN LC under the condition of $\theta=74^{\circ}$ (Hsu, 2007).

According to the above-mentioned results, Fig. 5 illustrates the cell configuration of the BHN LC. The BHN cell is composed of a DFLC doped with a suitable concentration of a chiral agent sandwiched between two indium-tin-oxide (ITO)-coated glass substrates covered with alignment films. To achieve necessary tilted-homeotropic molecular orientation with a proper pretilt angle, one can simply coat a homeotropic polyimide or a mixed solution of homeotropic- and planar-alignment polyimide on the substrates as the aligning layers with the treatment of mechanical rubbing on the top and bottom substrates in anti-parallel direction. While the adaptable range of the pretilt angle is very limited in a BHN LC cell with homeotropic alignment, cells with mixed alignment enable the tunable pretilt angle in a wider range by adjusting its composition (Yeung et al., 2006).

On the basis of the switching mechanism of the BHN LC (Hsu et al., 2007; Liang et al., 2008), a brief illustration, shown in Fig. 6, is provided to summarize the operation between the two stable states in a BHN LC cell. Here, $f_{1}$ and $f_{2}$ are the frequencies satisfying the conditions of $f_{1}<$ $f_{\mathrm{c}}$ and $f_{2}>f_{\mathrm{c}}$, and hence correspond to the positive and negative dielectric anisotropy $(\Delta \varepsilon)$ of the DFLC, respectively. The solid and dash lines represent the texture transitions from tT to $\mathrm{tH}$ and $\mathrm{tH}$ to $\mathrm{tT}$, respectively. Consider a $\mathrm{tT}$ state stabilized in the cell. The LC molecules will response to align perpendicular to the substrate when an electric field with frequency $f_{1}$ is applied vertically to the cell because the LC exhibits positive dielectric anisotropy $(\Delta \varepsilon>0)$. The molecules are then oriented homeotropically (i.e., vertically) in the cell at high voltage. This voltage-sustained state is called biased homeotropic $(\mathrm{bH})$ state. As the electric field is switched off, the molecules relax to the $\mathrm{tH}$ state with very high pretilt angle. Accordingly, the switching from $\mathrm{tT}$ to $\mathrm{tH}$ state follows a transition process of $\mathrm{tT}-\mathrm{bH}-\mathrm{tH}$. To switch the cell from $\mathrm{tH}$ to $\mathrm{tT}$ state, a connecting pulse with frequencies $f_{1}$ and $f_{2}$ is used. Note that the second pulse with 
frequency $f_{2}$ instantly follows the first one with frequency $f_{1}$. Once the connecting pulse is applied to the cell, the cell first induces the pulse with frequency $f_{1}$ which results in the switching of cell from $\mathrm{tH}$ to $\mathrm{bH}$ state. As the frequency of the connecting pulse changes from $f_{1}$ and $f_{2}$, where the dielectric anisotropy of the LC becomes negative $(\Delta \varepsilon<0)$, the dielectric coupling between the electric field and LC leads the molecules to align parallel to the substrate and induces flow effect. The cell is thus switched from bH state to another voltage-sustained state, called biased twisted (bT) state due to the backflow effect and applied electric field. The molecular in the bT state rotates about $2 \pi$ in the azimuthal angle. The bT state subsequently relax to the stable $\mathrm{tT}$ state as the driving pulse ends. Both $\mathrm{bT}$ and $\mathrm{tT}$ state have $2 \pi$-twist molecular orientation but the tilted angle in bT state is higher than that in the tT state. As a result, a transition process of $\mathrm{tH}-\mathrm{bH}-\mathrm{bT}-\mathrm{tT}$ is required within the switching from $\mathrm{tH}$ to $\mathrm{tT}$ state. It is worth reminding that the $\mathrm{tH}$ and $\mathrm{tT}$ states are of optical stability.

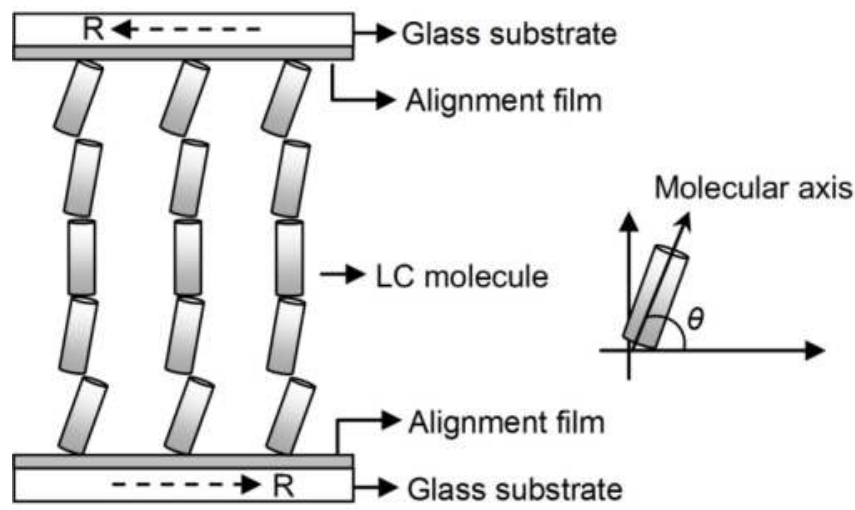

Figure 5. Schematic illustration of the configuration of a BHN LC cell. R, the rubbing direction.

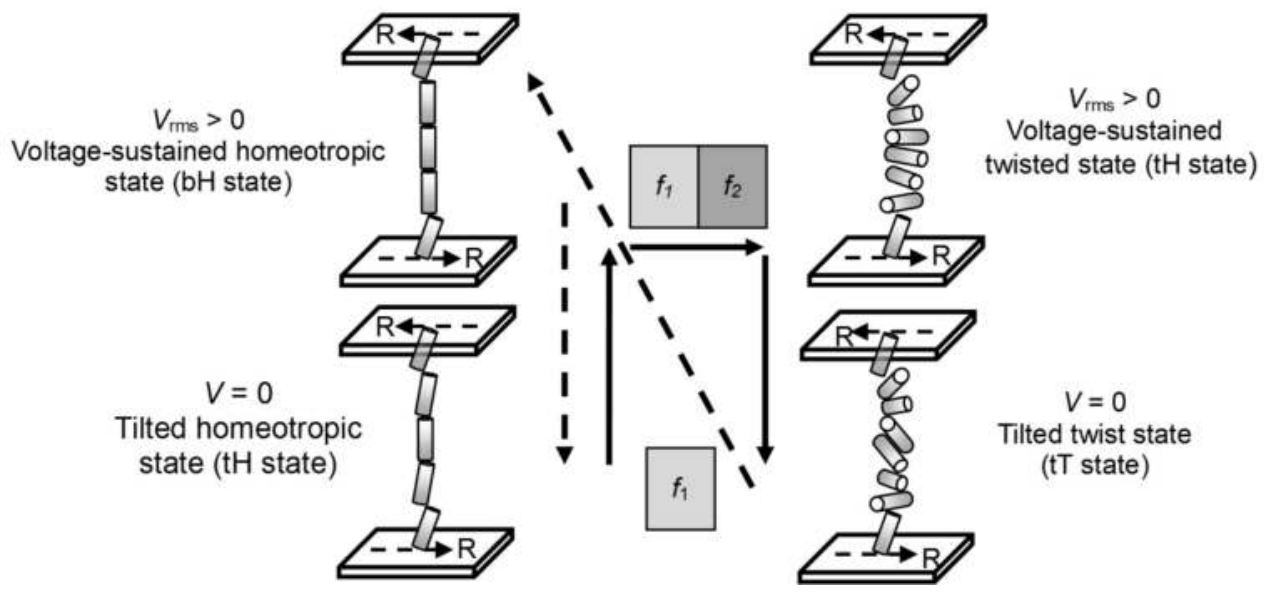

Figure 6. Bistable switching for a BHN LC cell upon the application of a frequency-modulated voltage pulse (adapted from Hsu et al., 2007). 


\subsection{Dual-frequency cholesteric liquid crystals}

The optical bistability is one of the unique features in cholesteric LC (CLC) and its applications in electro-optical devices (Bao et al., 2009; Berreman \& Heffner, 2006; Huang et al., 2003; Hsiao et al., 2011b; Xu \& Yang, 1997). CLCs, also termed chiral nematic LCs because of their structural nature to be the chiral versions of the nematic molecules, have been widely investigated in the literature. In a typical bistable CLC cell, the two stable states are the transparent planar $(\mathrm{P})$ state and light-scattering focal conic (FC) state. Switching between these two states can readily be achieved by adjusting the amplitude of applied electric field. For instance, when an electric field in square wave with a specific amplitude value is applied to the CLC cell, the texture will be changed from P to FC state. Further increasing the voltage over a critical value results in the transition of texture from FC to homeotropic $(\mathrm{H})$ state. The texture in the $\mathrm{H}$ state can be switched back to the $\mathrm{P}$ state by turning off the field rapidly or to the lightscattering FC state by turning off the field slowly. As a result, the P-to-FC transition can be accomplished directly by the external applied field whereas the FC-to-P transition is indirect since an intermediate $\mathrm{H}$ state is required. In addition, it takes a few seconds to relax from $\mathrm{H}$ to $\mathrm{P}$ state, meaning that the response time for the FC-to-P transition is quite slow. This drawback limits the CLC device for practical applications. In contrast to the conventional CLCs, the DFCLC device empowers direct two-way switching between two cholesteric states due to its material property of the frequency-revertible dielectric anisotropy and thus yields a feature of fast response speed (Hsiao et al., 2011b; Ma et al., 2010).

Figure 7 depicts the cell configuration of a typical CLC or DFCLC cell prepared in homogeneous (i.e., planar) alignment. A (DF)LC host doped with a chiral agent in proper concentration is sandwiched between two conductive glass substrates coated with planar alignment films. The rubbing directions of the top and bottom substrates are set to be antiparallel. As a consequence, a planar texture is initially formed, exhibiting periodic helix structure with its optical axis perpendicular to the substrate plane. Such a molecular orientation in the CLC cell itself can be regarded as a photonic crystal structure with its bandgap located within a designated wavelength regime.

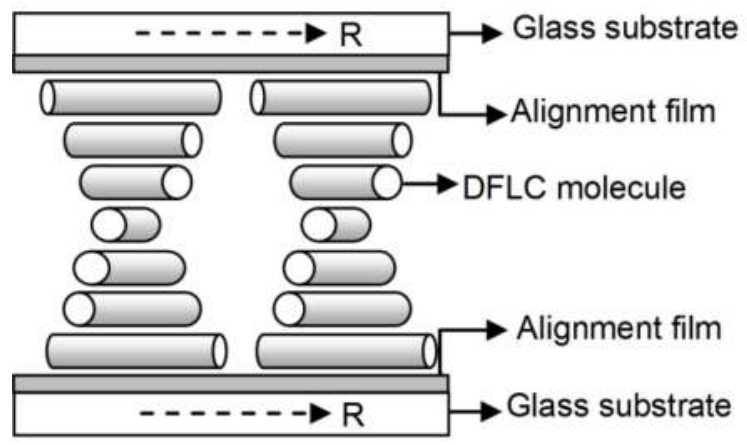

Figure 7. Schematic of the molecular configuration of a planar-alignment DFCLC cell. The helix axis is vertical in the initial state. 


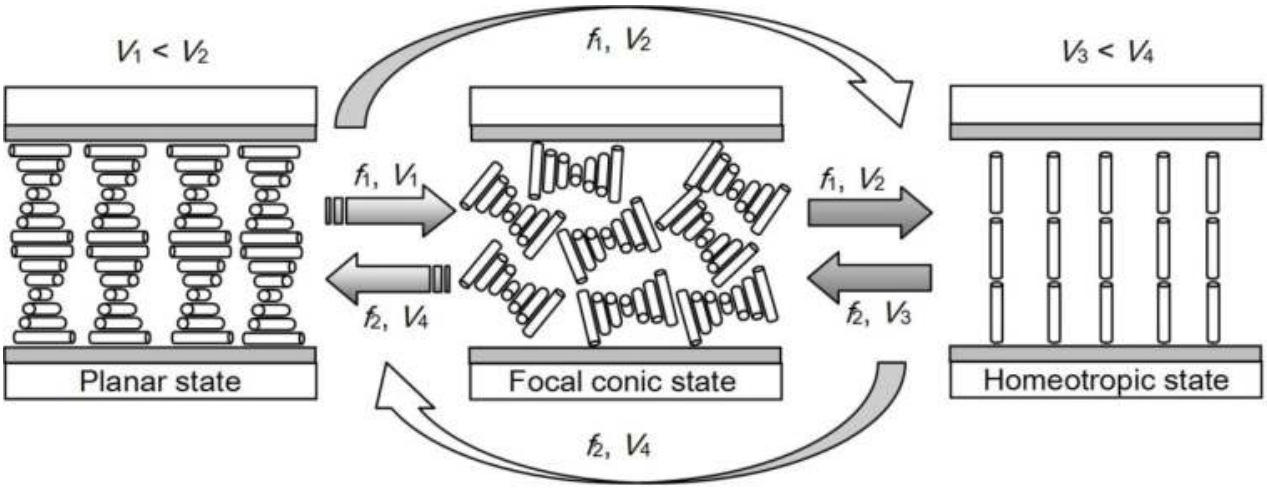

Figure 8. Operation principle and texture transitions of a DFCLC cell under the application of various voltage pulses (adapted from Hsiao et al., 2011a).

To clarity how the cholesteric textures are switched by frequency-modulated voltage pulses, Fig. 8 illustrates operations among three cholesteric states (i.e., P, FC, and H) in a DFCLC cell (Hsiao et al., 2011a). It should be noted here again that, when a voltage pulse is applied to the DFCLC cell, the dielectric anisotropy is positive at low frequencies $\left(f_{1}\right)$ and negative at high frequencies $\left(f_{2}\right)$ beyond the temperature-dependent crossover frequency $\left(f_{\mathrm{c}}\right)$. Once a voltage pulse with frequency $f_{1}$ is applied vertically to the DFCLC cell, the dielectric coupling leads the molecules to reorientation in parallel to the field direction and the helix tends to be unwound. Consequently, direct P-to-FC and P-to-H switchings can be induced at voltages $V_{1}$ and $V_{2}$, respectively. The switching mechanism of the DFCLC cell in the increasing-voltage process is the same as that of a conventional CLC one. It is worth mentioning here that while applying a pulse with frequency $f_{2}$, at which the molecules incline to align themselves homogeneously with respect to the substrate plane, the backward switching of the FC-to-P and H-to-P transitions can directly be accomplished at voltage $V_{3}$ and $V_{4}$, respectively. Noticeably, reversible direct-switching between FC and $\mathrm{H}$ state can also be achieved by individually applying a voltage pulse at voltage $V_{2}$ at $f_{1}$ and $f_{2}$. Note that $V_{2}$ is higher than $V_{1}\left(V_{2}>V_{1}\right)$ and $V_{4}$ is higher than $V_{3}\left(V_{4}>V_{3}\right)$ because switching between $\mathrm{P}$ and $\mathrm{H}$ states requires more energy to make the molecules reach balance. In the bistable DFCLC device, the P and FC textures are essentially the stable states whereas the $\mathrm{H}$ texture is a voltage-sustained state. Upon incorporating a certain content of photo-curable monomer (or prepolymer) into DFCLC, another type of CLC device called polymerstabilized cholesteric texture (PSCT), can be created after adequate polymerization. In such a PSCT cell, the stabilization of polymer network throughout the bulk cell enables the possession of stable $\mathrm{H}$ state (Ma et al., 2010). Combining the switching mechanism of DFCLC with PSCT, a novel tristable PSCT in which the texture can permanently be stabilized optically in the $\mathrm{P}, \mathrm{FC}$, and $\mathrm{H}$ states, has been demonstrated very recently (Hsiao et al., 2011a). While thinking about the use of the DFCLC in practical applications there are some issues that should be taken into consideration. The common drawback of the DFCLC is its considerable sensitivity to temperature. Typically, high operation voltage results in 
dielectric heating, which shifts the crossover frequency to a higher value (Yin et al., 2006; Wen \& Wu, 2005). On the other hand, a low-voltage pulse would be insufficient to induce the direct FC-to-P transition.

\section{Optical properties of one-dimensional photonic-crystal/memory-mode- liquid-crystal cells}

\subsection{Definition of the cell configuration}

A 1D PC structure incorporated with LC as a defect layer is well known to have the ability on tuning the optical characteristics such as light intensities and wavelengths of the defect modes. While most of investigations of 1D PC/LC cells are demonstrated based on DMLCs, this section is dedicated to a new classification of $1 \mathrm{D} \mathrm{PC/LC} \mathrm{cells} \mathrm{formed} \mathrm{by} \mathrm{infiltrating} \mathrm{with} \mathrm{a}$ MMLC as a defect layer. The configuration of a 1D PC/MMLC hybrid structure is schematically depicted in Fig. 9 (Hsiao et al., 2011c). Referring to the conventional 1D periodical structure, the 1D PC here is constructed with two identical dielectric mirrors having multilayer films deposited on the electrically conductive glass substrates. The MMLC, for example, the DFCLC whose electro-optical characteristic exhibits bistable or multistable switching, is infiltrated into the 1D PC as a defect layer with a thickness $d$. The dielectric multilayer consists of a number of high- and low-index materials stacked alternately.

To provide high reflectivity, the OPL of these two materials is theoretically set to be equal to one-quarter wavelength of the incident light, i.e., $n_{\mathrm{H}} d_{\mathrm{H}}=n_{\mathrm{L}} d_{\mathrm{L}}=\lambda / 4$, where $n_{\mathrm{H}}, n_{\mathrm{L}}$, and $d_{\mathrm{H}}, d_{\mathrm{L}}$ are the refractive indices and thicknesses of the high- and low-index materials in a multilayer, respectively, and $\lambda$ is the center wavelength in the PBG. Note that the reflectivity of the cell can further be enhanced by additionally coating a high-index film on the top of the multilayer. In other words, the total number of layers is odd (Hecht, 2002). The width of the PBG can be enlarged by increasing the refractive-index difference $\left(n_{\mathrm{H}}-n_{\mathrm{L}}\right)$ and shifting the central wavelength to redder regime. Figure 10 compares the trans-

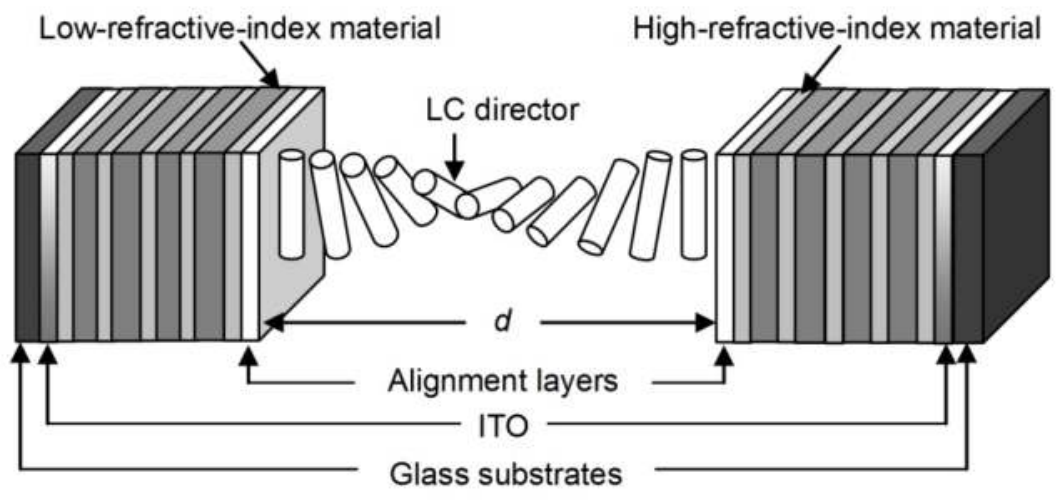

Figure 9. Cell configuration of a 1D PC/MMLC hybrid structure. The exemplary defect layer is a DFCLC (adapted from Hsiao et al., 2011c). 


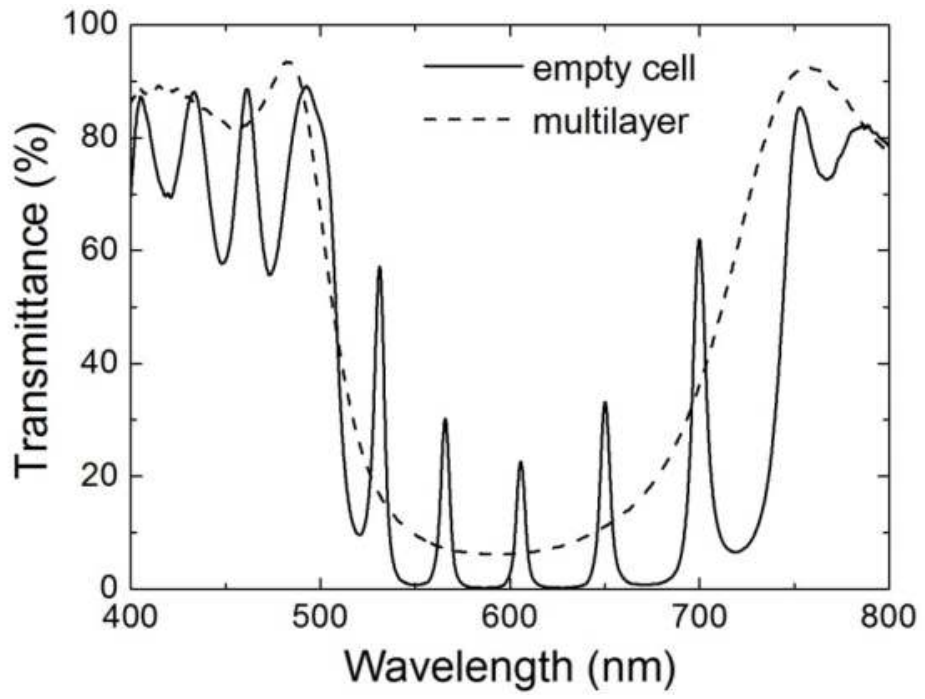

Figure 10. Transmittance of a dielectric mirror and an empty PC cell made of two identical dielectric mirrors.

mission spectra of a multilayer film on a glass substrate and a combination of two multilayer-coated substrates with a central air gap (empty cell). In this example, the multilayer film has nine layers, including five high-index layers and four low-index layers. Obviously, the width of PBG is around $250 \mathrm{~nm}$ with the central wavelength near $620 \mathrm{~nm}$ in the single multilayer. While sandwiching a defect layer between two multilayers, a number of defect modes are generated within the PBG. Note that the insertion of the defect layer does not essentially affect the width of the PBG. The profile of defect modes is influenced by some parameters. Ozaki and associates report that increasing the layer number in the multilayer decreases the line width and transmittance of defect-mode peaks because the Bragg-reflected light, dictated by the multilayer, is limited to reach the defect layer (Ozaki et al., 2004a). On the other hand, it is concluded by Zyryanov and coauthors that the number of defect modes is increased with increasing thickness of the defect layer or using the material with high refractive index as a defect layer (Zyryanov et al., 2008).

\subsection{One-dimensional photonic-crystal/bistable-chiral-tilted-homeotropic- nematic cells}

According to the operation of a BHN LC discussed in Section 2.1, applying an external electric field to the PC/BHN hybrid cell enables the switching among four specific states; i.e., $\mathrm{tH}, \mathrm{bH}, \mathrm{tT}$, and bT states. Due to different molecular orientation of LC as well as the contributions of effective refractive index ( $n_{\text {eff }}$ ), the transmission spectra of the 1D PC/BHN hybrid structure in four different states without employing any polarizer disclose dissimilar spectral profiles, as shown in Fig. 11. Recalling the mathematical expression of the effective refractive index of an optically uniaxial material (Yeh \& Gu, 1999b) 


$$
\mathrm{n}_{\text {eff }}=\frac{\mathrm{n}_{\mathrm{o}} \mathrm{n}_{\mathrm{e}}}{\left(\mathrm{n}_{\mathrm{o}} \cos ^{2} \theta+\mathrm{n}_{\mathrm{e}} \sin ^{2} \theta\right)^{1 / 2}}
$$

where $n_{\mathrm{o}}$ and $n_{\mathrm{e}}$ are the ordinary and extraordinary refractive indices of the LC, respectively and $\theta$ is the tilted angle of LC molecules measured from the substrate plane, one can comprehend that the defect modes in the $\mathrm{bH}$ state are attributed by sole $n_{\mathrm{o}}$ because most of LC molecules in this state are aligned normal to the substrate $\left(\theta=90^{\circ}\right)$. In contrast, the molecules in the tH state are oriented at a high tilted angle with respect to the substrate plane whereas both the tT and bT state have a $2 \pi$-twist molecular orientation. Consequently, the defect modes have more peaks in all of the other three states due to the contribution of $n_{\mathrm{e}}$ to the resulting $n_{\text {eff, }}$ which is larger than $n_{\mathrm{o}}$.

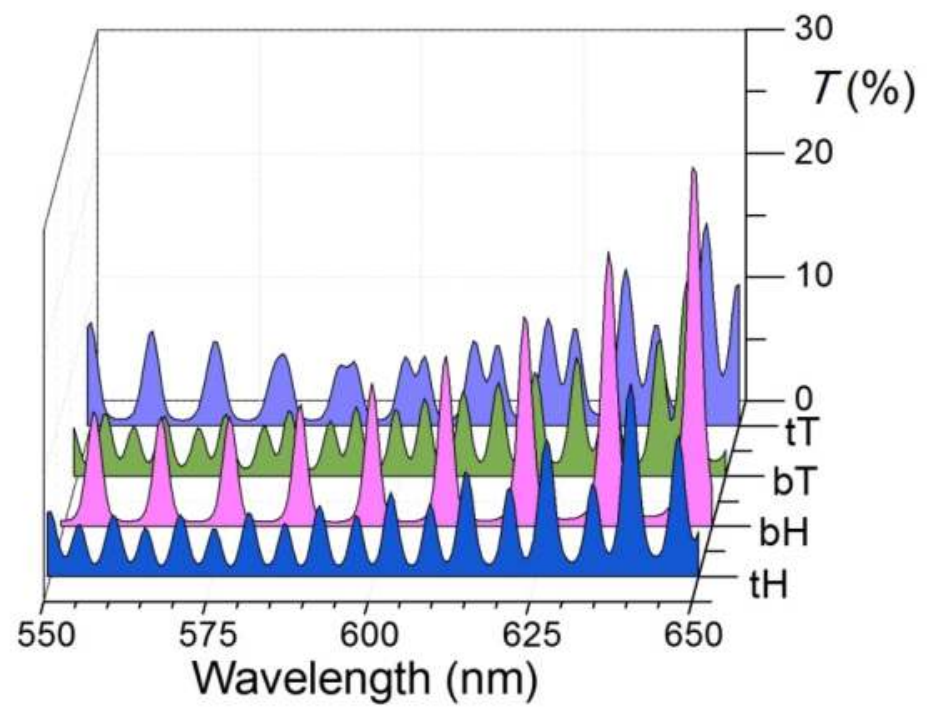

Figure 11. Transmission spectra of the four states of the PC/BHN LC cell within the photonic bandgap.

Similar to the tunable mechanism in the 1D PC/DMLC device, the tunability in defect modes of the PC/BHN cell can be realized by dynamic switching between two proper states. In the case of two homeotropic states, Fig. 12 shows defect modes of the $\mathrm{tH}$ and bH state in specific wavelength range without employing any polarizers. In the $\mathrm{tH}$ state, the peaks overlapped with those in the bH state characterizes the ordinary defect modes whereas other peaks are explained as the extraordinary defect modes. According to Eq. (2), the effective refractive index in the $\mathrm{tH}$ state is higher than that in the $\mathrm{bH}$ state, determined by their molecular orientation. In addition, it can also be conceived that operation between th to bH state enables the control on the contribution of extraordinary index as well as the effective refractive index by the applied voltage. Accordingly, the transmission-intensity tunability in the extraordinary defect modes can be achieved by switching between these two states. For instance, the switching between $\mathrm{tH}$ and bH state is created by applying an electric field at 
low frequency $f_{1}$ (see Section 2.1). Considering a forward switching from tH to bH state, the intensity of peaks in the extraordinary defect modes is reduced with increasing voltage due to the decrease in the effective refractive index. The intensity then reaches minima as the $t H$ state transfers completely to the bH state. Similarly, backward switching from bH to tH state reproduces the original intensity of peaks.

Furthermore, Fig. 13 reveals the wavelength tunability of the defect modes in the bT state of the PC/BHN cell. Switching to the bT state is accomplished from bH state by adjusting the frequency from low frequency $f_{1}$ to high frequency $f_{2}$ (see Section 2.1). In the bT state, the tilted angle of molecular director is higher than that in the stable tT state, as illustrated in Fig. 6 . Thus, while increasing the high-frequency voltage, the tilted angle is reduced and increases the contribution of $n_{\mathrm{e}}$ component to the overall effective refractive index. As a result, redshift (Miroshnichenko et al., 2008a, 2008b; Zyryanov et al., 2008a) of the defect modes as a function of increasing voltage is observed, demonstrating the ability of defect mode tunability.

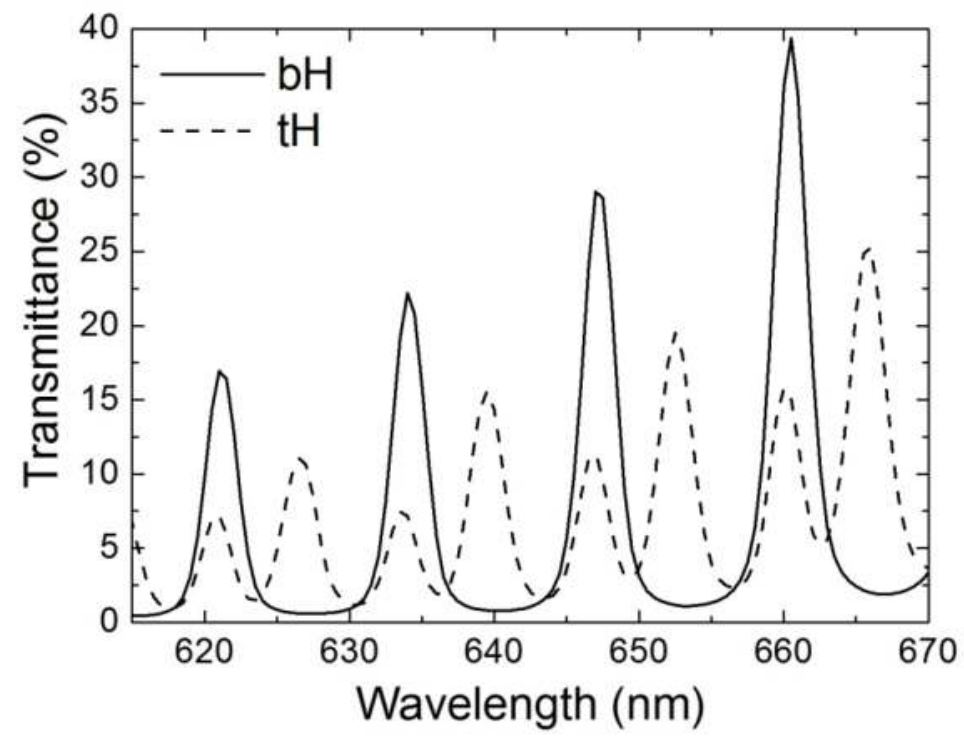

Figure 12. Comparison of defect modes in the transmission spectra for the PC/BHN LC cell in $\mathrm{tH}$ and bH state.

\subsection{One-dimensional photonic-crystal/dual-frequency-cholesteric-liquid-crystal cells}

The texture PC/DFCLC cell can be switched to three states, according to its operation mechanism illustrated in Fig. 7. These textures are P, FC, and $\mathrm{H}$ state. While the $\mathrm{P}$ and FC can be the stable states $\mathrm{H}$ state is the voltage-sustained state. Figure 14 shows the transmission spectra of the PC/DFCLC cell in two stable states. The cell in the transparent $\mathrm{P}$ state reveals a number of defect modes with transmission intensity around 25 to $60 \%$ in the 
PBG. When the texture is switched from $\mathrm{P}$ to FC state by a voltage pulse of $V_{1}=20 \mathrm{~V}_{\text {rms }}$ at $f_{1}=$ $1 \mathrm{kHz}$, the intensity of defect modes drops dramatically to about $1 \%$ due to the formation of randomly oriented poly-domain in this state. Accordingly, the bistble switching between $\mathrm{P}$ and FC state results in the tunable defect modes between turning on and off state that enable the PC/DFCLC to serve as an electrically tunable light filter with features of fast switching speed and low power consumption. When the cell is switched between $\mathrm{P}$ and $\mathrm{H}$ state the tunability on the wavelength of the defect modes can be realized.

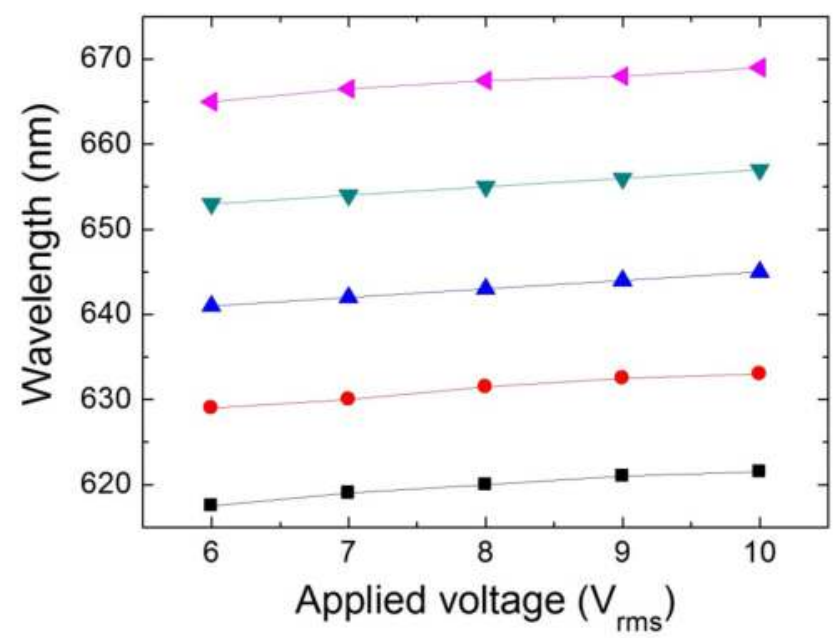

Figure 13. Redshift of the defect modes in the bT state with increasing voltage (adapted from Wu et al., 2011).

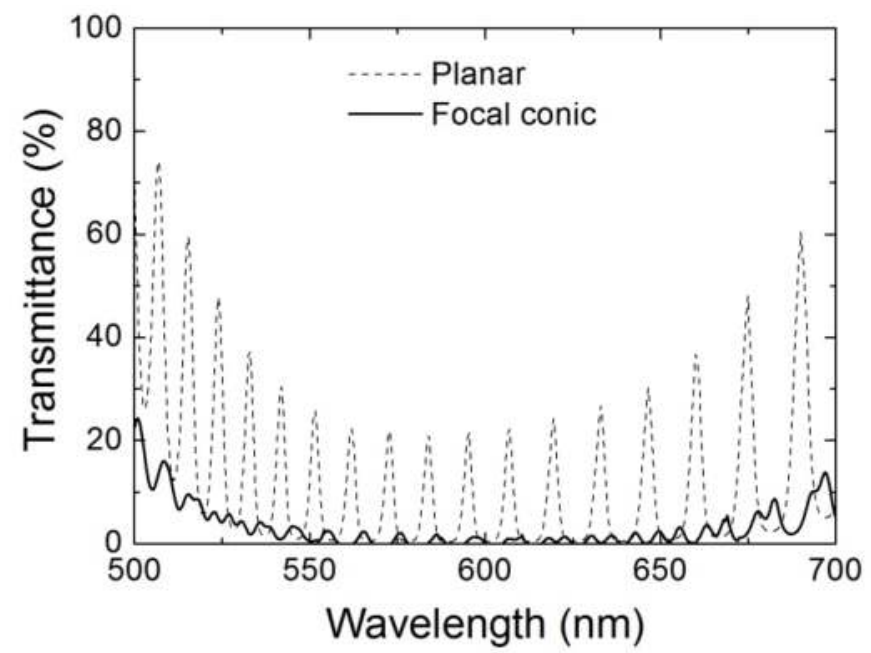

Figure 14. Transmission spectra of a PC/DFCLC in the photonic bandgap in two stable states (adapted from Hsiao et al., 2011c), demonstrating light-intensity tunability. 
Figure 15 shows the transmission spectra of the PC/DFCLC cell in the P and H state. It can clearly be recognized that two separated sets of defect modes with comparable transmission strengths are obtained. In contrast to the defect modes in the P state, the defect modes in the $\mathrm{H}$ state shifts to the shorter wavelengths due to the decrease in the effective refractive index. Noticeably, the peaks of defect modes in one of the two states overlaps to the stop band in the other state indicating the complementary nature in wavelengths. Furthermore, Fig. 16 demonstrates a new approach for tuning the peak transmittance of the defect modes by varying the frequency of the applied voltage pulse. In this example, it is noted that the dielectric anisotropy of the DFLC is negative and it increases with increasing frequency in the frequency range from 20 to $100 \mathrm{kHz}$. Therefore, while a $20 \mathrm{kHz}$ voltage pulse align the molecules in the FC state the cell subsequently transits to the planar state as the frequency increases due to the enhancement in the torque of dielectric coupling. As a result, the transmittance of peaks in the defect modes increases with increasing frequency due to the change of molecular orientation.

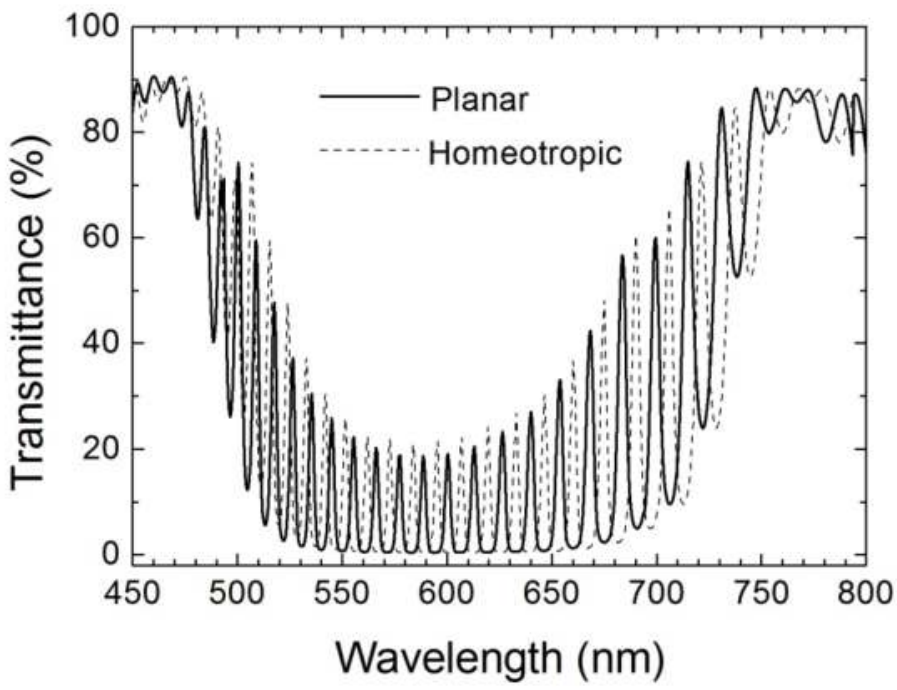

Figure 15. Transmittance of the PC/DFCLC in the photonic bandgap with two different sets of defect modes in the $\mathrm{P}$ and $\mathrm{H}$ states (adapted from Hsiao et al., 2011c).

\section{Memorable multichannel devices based on one-dimensional photonic- crystal/memory-mode-liquid-crystal cells}

\subsection{Bistable one-dimensional photonic-crystal/chiral-tilted-homeotropic-nematic cells under parallel polarizers}

It is clarified from Section 3.2 that the electrically tunable defect modes in a $1 \mathrm{D}$ PC/BHN hybrid cell can be realized even for the light propagating through the cell without employing any polarizers. For instance, the transmittance tunabiltiy in the extraordinary 
defect modes is performed in the cell while switching between the $\mathrm{tH}$ and bH states, suggesting a potential application in light filter. Moreover, operation in bT state as a function of increasing voltage results in the tunable wavelength, shifting to the longer wavelengths, in the defect modes. Although above mentioned mechanisms enable the $\mathrm{PC} / \mathrm{BHN}$ to extent its use in various optical devices without employing any polarizers, all of them are performed by the dynamic switching between one stable state and voltagesustained state. Here, a concept of wavelength-tunability in defect modes, characterized by the two stable states of the PC/BHN cell is proposed by setting the cell between two parallel polarizers (meaning linear polarizers with parallel transmission axes). Figure 17 depicts the transmission spectra of the PC/BHN cell in stable states with and without parallel polarizers. The angle between the rubbing direction of the cell and the transmission axis of either polarizer is denoted as $\beta$. While the incoming light passing through first polarizer at $\beta=0^{\circ}$ represents as E-ray, it becomes O-ray at $\beta=90^{\circ}$. It is known from Section 3.2 that the effective refractive index in the cell without any polarizers is contributed by both $n_{\mathrm{o}}$ and $n_{\mathrm{e}}$; thus, forming minute defect modes in both the $\mathrm{tH}$ and $\mathrm{tT}$ states, as shown in Fig. 17(a). Along with pervious investigations of the spectral properties of 1D PC/LC cells with crossed polarizers (Lin et al., 2010; Zyryanov et al., 2010), except for the homeotropic state, the o-ray and e-ray propagating through the LC bulk senses $n_{\mathrm{o}}$ and $n_{\mathrm{e}}$, respectively. Accordingly, when the cell is set between parallel polarizers with polarization angles of $90^{\circ}$ and $0^{\circ}$, the defect modes associated with $n_{\mathrm{o}}$ and $n_{\mathrm{e}}$ are discriminative, respectively, as shown in Figs. 17(b) and (c). In addition, it is noticeable that two divided sets of defect modes corresponding to the two stable states are observed in the cell with parallel polarizers. This result implies a concept for multichannel applications, characterized by the optically bistable states.

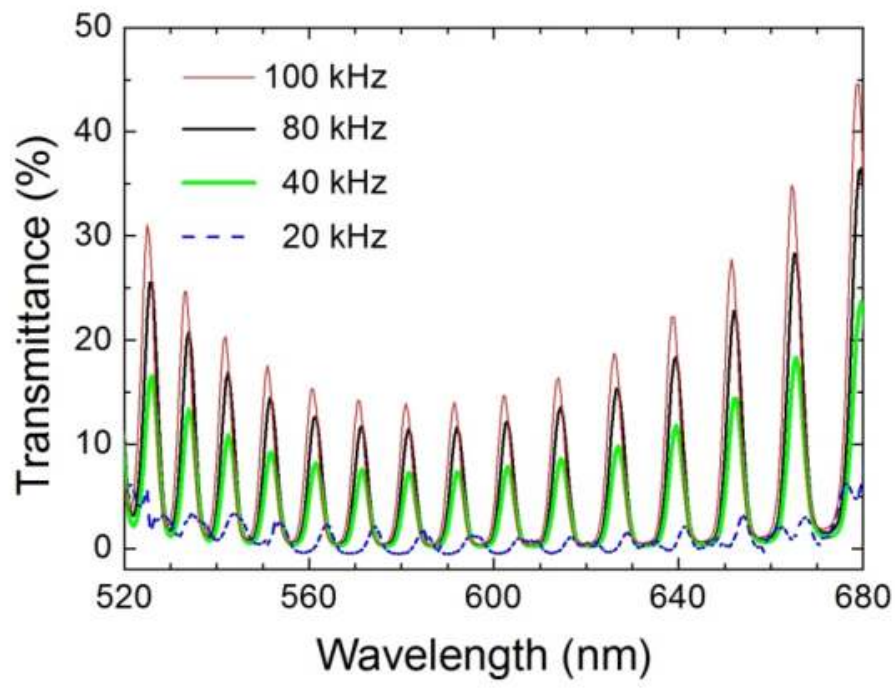

Figure 16. Transmittance of stable defect modes in the PBG induced by a 24.5-V voltage pulse at various frequencies. 


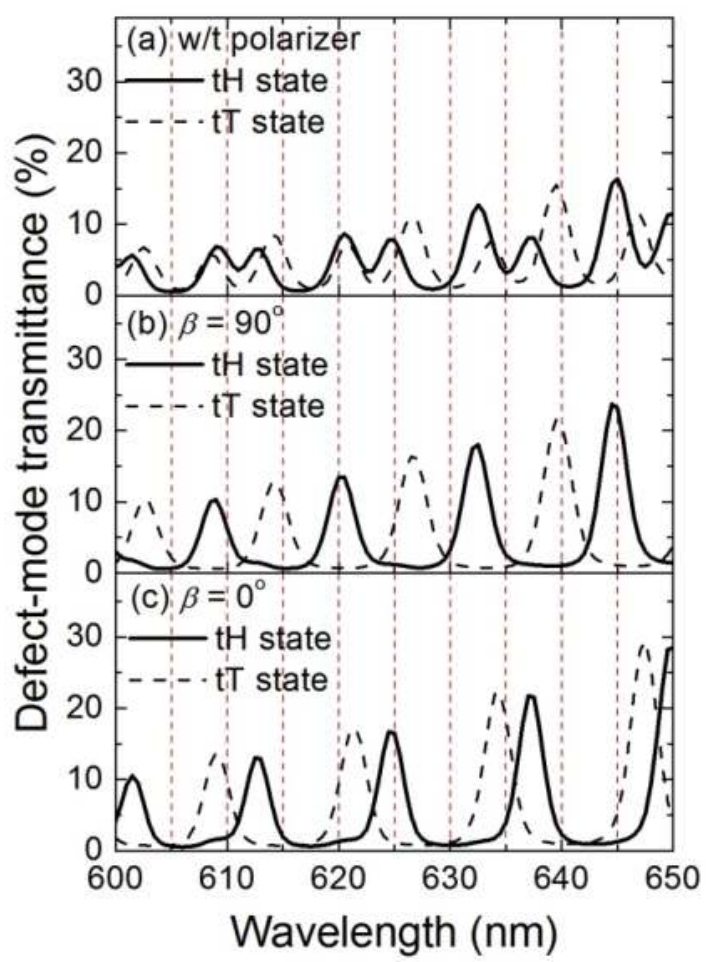

Figure 17. Transmittance of a PC/BHN cell (a) without polarizers and with parallel polarizers at (b) $\beta=$ $90^{\circ}$ and (c) $\beta=0^{\circ}$.

\subsection{Tristable one-dimensional photonic-crystal cell with polymer-stabilized cholesteric textures}

Based on the cell configuration of the DFCLC cell, a tristable PSCTs cell is created by incorporating photo-curable monomers into DFCLC with proper amounts due to the distribution of polymer networks throughout the cell. These three PSCTs are also referred to as the $\mathrm{P}, \mathrm{FC}$, and $\mathrm{H}$ states. According to the operation principle illustrated in Fig. 8, switching from one to another state in the PSCTs can be achieved by applying suitable frequency-modulated voltage pulses. In contrast to the electrical tunability mechanisms in the 1D PC/DFCLC cell, the 1D PC structure infiltrated with tristable PSCTs is expected to characterize the tunability on intensity and wavelength of defect modes by its tristable states. Figure 18 demonstrates the transmission spectra of the 1D PC/PSCTs cell in three stable states. Since the spectra profiles in these three states is revealed in Section 3.3, it can be understood from Fig. 18 that the mechanisms of tunable intensity and wavelength in the defect modes can also be performed in the PC/PSCT cell by operating between two stable states. In the case of intensity tunability, the FC state is certainly represented as the light off state because the transmission of defect modes in this state is the lowest. While switching 
the cell from FC to either P or H state, the transmission of defect modes becomes intense, denoting the light on state. It is emphasized that all of the three states can be stabilized permanently after voltage pulse removal. On the other hand, the position of defect modes can be shifted by switching between the $\mathrm{P}$ and $\mathrm{H}$ states. The appearance of two individual sets of defect modes, characterized by the $\mathrm{P}$ and $\mathrm{H}$ states in the cell, is applicable for enhancing the performance of the multichannel device. While the transmission of the peaks in defect modes can be adjusted by frequency-modulated voltage pulses with fixed amplitude, Fig. 19 demonstrates that the transmittance-modulation in the defect modes is performed by modulating the amplitude of voltage pulse at fixed frequency. In this case, the initial state of the cell is $\mathrm{H}$ state and the frequency is $f_{2}=100 \mathrm{kHz}$, corresponding to the negative dielectric anisotropy of the DFLC. Accordingly, the PC/PSCTs cell is transferred from the initial $\mathrm{P}$ state to $\mathrm{H}$-to-FC mixed state and FC state when the voltage increases from 0 to $40 \mathrm{~V}$. If the initial state is $\mathrm{P}$ state, it is comprehensible that the transmission tunability can be achieved by varying voltage at frequency $f_{1}$. As a result, this powerful photonic device has potential to extend the application, permitting its use as an electrically controllable and optically tristable multichannel filter without requiring any polarizers.

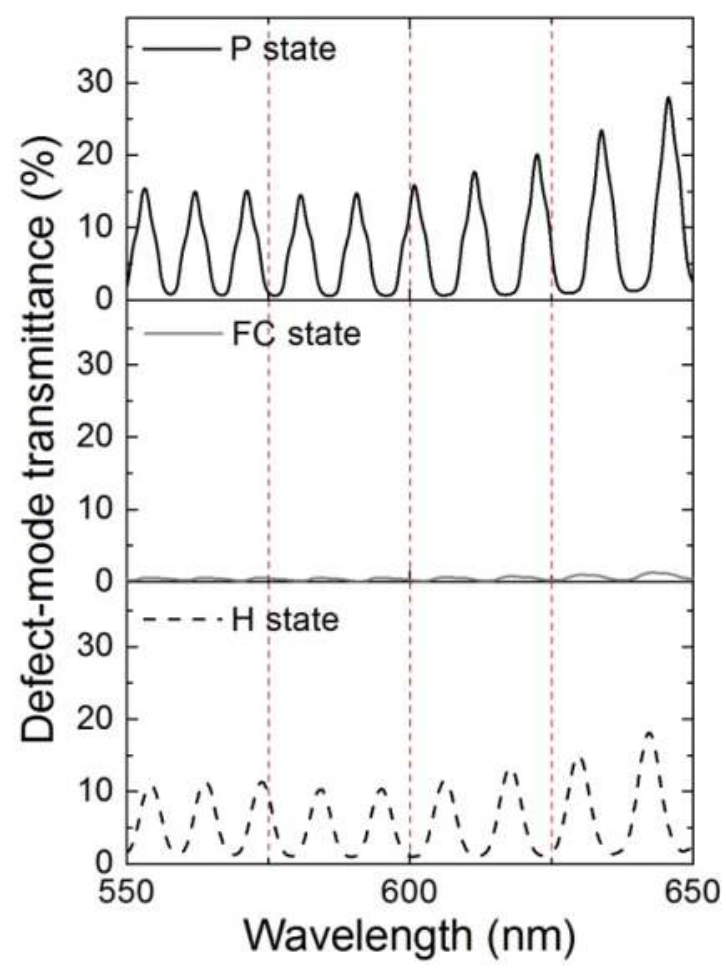

Figure 18. Transmission within the photonic bandgap of a PC/DFCLC cell in three stable states (adapted from Hsiao et al., 2011a). 


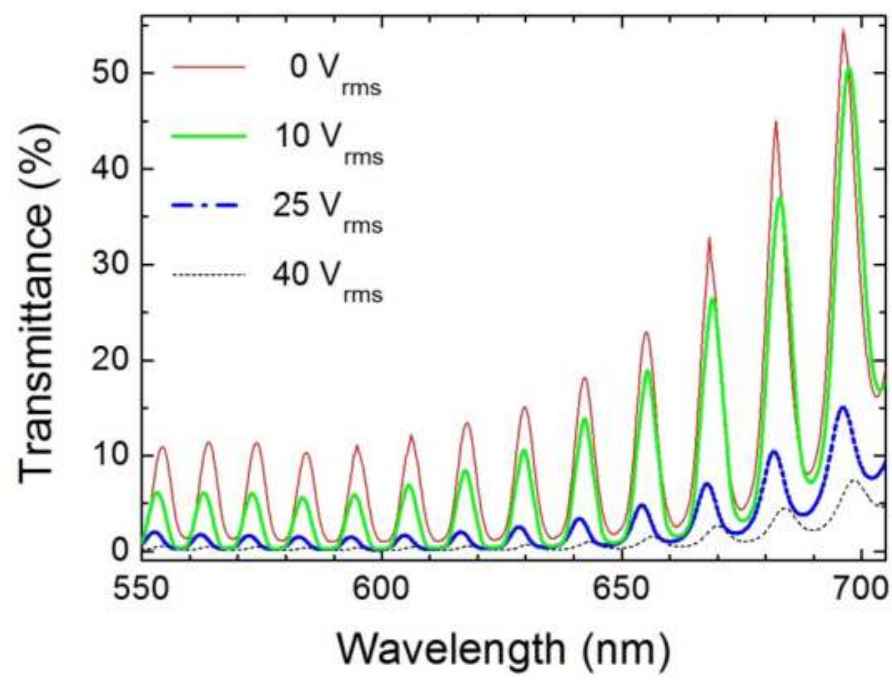

Figure 19. Transmittance of stable defect modes in the photonic bandgap induced by a voltage pulse at frequency $f_{1}$ with various amplitudes, (adapted from Hsiao et al., 2011a). Note that the texture in the cell is transited from planar to focal conic state with increasing voltage.

\section{Conclusions}

In this chapter, two types of 1D PC/MMLC cells which exhibit both electrical tunability and optical bi- or tri-stability in the defect modes have been reviewed in accordance with our previously published papers. Several fascinating features have also been keynoted. In the case of the PC/BHN cell based on a chiral-agent-doped DFLC infiltrated as a defect layer, it can be switched in four different states by applying voltage pulses with designated waveforms. The dynamic switching in the voltage-sustained bT state results in the redshift of the defect modes with increasing voltage (at $100 \mathrm{kHz}$ ) due to the increase in the effective refractive index. Moreover, the tunable defect modes in the PC/BHN cell can be achieved by their two stable states ( $\mathrm{bH}$ and bT) when the cell is set between parallel polarizers. Such bifunctional photonic devices pave a new pathway for the application in low-powerconsumption multichannel optical switches and integrated photonic devices. On the other hand, switching among the P, FC, and H states in a PC/DFCLC cell can be regulated rapidly, directly and reversibly by using frequency-modulated voltage pulses. The ability of wavelength tunability of defect modes in the PC/DFCLC cell is achieved by the texture transition from the stable $\mathrm{P}$ state to the voltage-sustained $\mathrm{H}$ state. Owing to the frequencydependent dielectric anisotropy of the DFLC, the transmittance of the defect modes can be tuned by both the voltage frequency and amplitude, providing a new way for intensity tunability through such a filter characterized by the defect modes. This device requires no polarizers and is of low power consumption. Noticeably, the voltage-sustained H state in the bistable DFCLC can further be a third optically stable state by incorporating an adequate amount of photo-curable monomer, oligomer, or prepolymer into the DFCLC material to 
create polymer networks in the defect layer. Such a DFCLC cell exhibiting three stable states $-\mathrm{P}, \mathrm{FC}$, and $\mathrm{H}-$ is known as a tristable PSCT. Referring to the aforementioned tunability in the PC/DFCLC, the wavelength tunability and transmittance tunability of defect modes in the PC/PSCT cell can be achieved - the intensities of the defect modes can be regulated by the amplitude of voltage in the mixed states and the wavelengths be switched by the frequency in the $\mathrm{H}$ and $\mathrm{P}$ states. The cell can directly be switched from one to another stable state by employing a proper frequency- modulated voltage pulse on the cell.

1D PC/LC has received much attention in recent years due to its tunability in defect modes within the PBG. It has been established that this hybrid PC structure enables the control over the defect modes by electric field, magnetic field, and the like as a stimulus. The resulting features make it applicable for designing tunable photonic devices such as a multichannel filter, light shutter, and optical modulator. Specifically, incorporating MMLC as a defect layer in the 1D PC provides both the optically memorable and tunable defect modes characterized by the stable states, allowing the device to be of low power consumption. Such a 1D PC/MMLC device thus extends its use for green products.

\section{Author details}

Po-Chang $\mathrm{Wu}$

Department of Physics, Chung Yuan Christian University, Chung-Li, Taiwan, Republic of China

Wei Lee

Department of Physics, Chung Yuan Christian University, Chung-Li, Taiwan, Republic of China College of Photonics, National Chiao Tung University, Guiren Dist., Tainan, Taiwan,

Republic of China

\section{Acknowledgement}

The authors acknowledge the financial support from the National Science Council of the Republic of China (Taiwan) under grant Nos. NSC 98-2923-M-033-001-MY3 and NSC 982112-M-009-023-MY3, and are grateful to Yu-Cheng Hsiao, Yu-Ting Lin, Ivan Timofeev, Chong-Yin $\mathrm{Wu}$, Yi-Hong Zou, and Victor Ya. Zyryanov for their assistance with the preparation of this manuscript.

\section{References}

Arkhipkin, V. G.; Gunyakov, V. A.; Myslivets, S. A.; Gerasimov, V. P.; Zyryanov, V. Ya.; Vetrov, S. Ya. \& Shabanov, V. F. (2008). One-Dimensional Photonic Crystals with a Planar Oriented Nematic Layer: Temperature and Angular Dependence of the Spectra of Defect Modes. Journal of Experimental and Theoretical Physics, Vol. 106, No. 2, pp. 388398 
Arkhipkin, V. G.; Gunyakov, V. A.; Myslivets, S. A.; Zyryanov, V. Ya. \& Shabanov, V. F. (2007). Angular Tuning of Defect Modes Spectrum in the One-Dimensional Photonic Crystal with Liquid-Crystal Layer. European Physical Journal E, Vol. 24, No. 3, pp. 297-302

Bao, R.; Liu, C.-M. \& Yang, D.-K. (2009). Smart Bistable Polymer Stabilized Cholesteric Texture Light Shutter. Applied Physics Express, Vol. 2, pp. 112401

Berreman, D. W. \& Heffner, W. R. (1980). New Bistable Cholesteric Liquid-Crystal Display. Applied Physics Letters, Vol. 37, No. 1, pp. 109-111

Blanco, A.; Chomski, E.; Grabtchak, S.; Ibisate, M.; John, S.; Leonard, S. W.; Lopez, C.; Meseguer, F.; Miguez, H.; Mondia, J. P.; Ozin, G. A.; Toader, O. \& van Driel, H. M. (2000). Large-Scale Synthesis of a Silicon Photonic Crystal with a Complete ThreeDimensional Bandgap near 1.5 Micrometers. Nature, Vol. 405, No. 6785, pp. 437-440

Bobrovsky, A.Yu.; Boiko, N. I.; Shibaev, V. P. \& Wendorff, J. H. (2003). Cholesteric Mixtures with Photochemically Tunable Circularly Polarized Fluorescence. Advance Materials, Vol. 15, No. 4, pp. 282-287

Choi, S. S.; Morris, Stephen M.; Huck, Wilhelm T. S. \& Coles, H. J. (2009). Electrically Tuneable Liquid Crystal Photonic Bandgaps. Advance Materials, Vol. 21, No. 38, pp. 3915-3918

Chow, E.; Lin, S. Y.; Johnson, S. G.; Villeneuve, P. R.; Joannopoulos, J. D.; Wendt, J. R.; Vawter, G. A.; Zubrzycki, W.; Hou, H. \& Alleman, A. (2000). Three-Dimensional Control of Light in a Two-Dimensional Photonic Crystal Slab. Nature, Vol. 407, No. 6807, pp. 983-986

Fleming, J. G. \& Lin. S.-Y. (1999). Three-Dimensional Photonic Crystal with a Stop Band from 1.35 to $1.95 \mu \mathrm{m}$, Optics Express, Vol. 24 No. 1, pp. 49-51

Gooch, C. H. \& Tarry, H. A. (1975). The Optical Properties of Twisted Nematic Liquid Crystal Structures With Twist Angles < 90 Degrees. Journal Physics D Applied Physics, Vol. 8, No. 13, pp. 1575-1584

Hecht., E. (2002). Applications of Single and Multilayer Films. In: Optics, Ch. 8, pp. 425-431, Addison Wesley, ISBN 0-321-18878-0, San Francisco

Hsiao, Y.-C.; Hou, C.-T.; Zyryanov, V. Ya. \& Lee, W. (2011a). Multichannel Photonic Devices Based on Tristable Polymer-Stabilized Cholesteric Textures. Optics Express, Vol. 19, No. 8, pp. 7349-7355

Hsiao, Y.-C.; Tang, C.-Y. \& Lee, W. (2011b). Fast-Switching Bistable Cholesteric Intensity Modulator. Optics Express, Vol. 19, No. 10, pp. 9744-9749

Hsiao, Y.-C.; Wu, C.-Y.; Chen, C.-H.; Zyryanov, V. Ya. \& Lee, W. (2011c). Electro-Optical Device based on Photonic Structure with a Dual-Frequency Cholesteric Liquid Crystal. Optics Letters, Vol. 36, No. 14, pp. 2632-2634

Hsu, J.-S. (2007). Stability of Bistable Chiral-Tilted Homeotropic Nematic Liquid Crystal Displays. Japanese Journal of Applied Physics, Vol. 46, No. 11, pp. 7378-7381

Hsu, J.-S.; Liang, B.-J. \& Chen, S.-H. (2004). Bistable Chiral Tilted-Homeotropic Nematic Liquid Crystal Cells. Applied Physics Letters, Vol. 85, No. 23, pp. 5511-5513.

Hsu, J.-S.; Liang, B.-J. \& Chen, S.-H. (2007). Dynamic Behaviors of Dual Frequency Liquid Crystals in Bistable Chiral Tilted-Homeotropic Nematic Liquid Crystal Cell. Applied Physics Letters, Vol. 89, No. 5, pp. 091520 
Huang, C. Y.; Fu, K.-Y.; Lo, K.-Y. \& Tsai, M.-S. (2003). Bistable Transflective Cholesteric Light Shutters. Optics Express, Vol. 11, No. 6, pp. 560-565

Huang, Y.; Zhou, Y.; Doyle, C. \& Wu, S.-T. (2006). Tuning the Photonic Band Gap in Cholesteric Liquid Crystals by Temperature-Dependent Dopant Solubility. Optics Express, Vol. 14, No. 3, pp. 1236-1244

Imada, M.; Noda, S.; Chutinan, A.; Tokuda, T.; Murata, M. \& Sasaki, G. (1999). Coherent Two-Dimensional Lasing Action in Surface-Emitting Laser with Triangular-Lattice Photonic Crystal Structure. Applied Physics Letters, Vol. 75, No. 3, pp. 316-318

Jhun, C. G.; Chen, C. P.; Lee, U. J.; Lee, S. R.; Yoon, T. H. \& Kim, J. C. (2006). Tristable Liquid Crystal Display with Memory and Dynamic Operating Modes. Applied Physics Letters, Vol. 89, No. 12, pp. 123507

John, S. (1987). Strong Localization of Photons in Certain Disordered Dielectric Superlattices. Physical Review Letters, Vol. 58, No. 23, pp. 2486-2489

Kasano M.; Ozaki, M.; Yoshino, K.; Ganzke, D. \& Haase, W. (2003). Electrically Tunable Waveguide Laser based on Ferroelectric Liquid Crystal. Applied Physics Letters, Vol. 82, No. 23, pp. 4026-4028

Knight, J. C.; Broeng, J;. Birks, T. A. \& Russell, P. St. J. (1998). Photonic Band Gap Guidance in Optical Fibers. Science, Vol. 282, No. 5393, pp. 1476-1478

Knight, Jonathan C. (2003). Photonic Crystal Fibres. Nature, Vol. 424, No. 6950, pp. 24862589.

Krauss, Thomas F.; De La Rue, Rchard M. \& Brand, S. (1996). Two-Dimensional PhotonicBandgap Structures Operating at Near-Infrared Wavelengths. Nature, Vol. 383, No. 6602, pp. 699-702

Liang B.-J. \& Lin, C.-L. (2007). Crucial Influence on $d / p$ Range in Bistable Chiral TiltedHomeotropic Nematic Liquid Crystal Cells. Journal of Applied Physics, Vol. 102, No. 12, pp.124504

Liang, B.-J.; Hsu, J.-S.; Lin, C.-L. \& Hsu, W.-C. (2008). Dynamic Switching Behavior of Bistable Chiral-Tilted Homeotropic Nematic Liquid Crystal Displays. Journal of Applied Physics, Vol. 104, No. 7, pp. 074509

Lin, T.-H.; Chen, Y.-J.; Wu, C.-H.; Fuh, Andy Y.-G.; Liu, J.-H. \& Yang, P.-C. (2005). Cholesteric liquid crystal laser with wide tuning capability. Applied Physics Letters. Vol. 86 pp. 161120

Lin, T.-H.; Jau, H.-C.; Chen, C.-H.; Chen, Y.-J.; Wei, T.-H.; Chen, C.-W. \& Fuh, Andy Y.-G. (2006). Electrically Controllable Laser based on Cholesteric Liquid Crystal with Negative Dielectric Anisotropy. Applied Physics Letters, Vol. 88, No. 6, pp. 061122

Lin, Y.-T.; Chang, W.-Y.; Wu, C.-Y.; Zyryanov, V. Ya. \& Lee, W. (2010). Optical Properties of One-Dimensional Photonic Crystal with a Twisted-Nematic Defect Layer. Optics Express, Vol. 18, No. 26, pp. 26959-26964

Ma, J.; Shi, L. \& Yang, D.-K. (2010). Bistable polymer stabilized cholesteric texture light shutter. Apply Physics Express, Vol. 3, No. 2, pp. 021702

Matsuhisa, Y.; Ozaki, R.; Takao, Y. \& Ozaki, M. (2007). Linearly Polarized Lasing in OneDimensional Hybrid Photonic Crystal containing Cholesteric Liquid Crystal. Journal of Applied Physics, Vol. 101, No. 3, pp. 033120 
Matsuhisa, Y.; Ozaki, R.; Yoshino, K. \& Ozaki, M. (2006). High Q Defect Mode and Laser Action in One-Dimensional Hybrid Photonic Crystal containing Cholesteric Liquid Crystal. Applied Physics Letters, Vol. 89, No. 10, pp. 101109

Miroshnichenko A. E.; Brasselet, E. \& Kivshar, Y. S. (2008a). All-Optical Switching and Multistability in Photonic Structures with Liquid Crystal Defects. Applied Physics Letters, Vol. 92, No. 25, pp. 253306.

Miroshnichenko A. E.; Brasselet, E. \& Kivshar, Y. S. (2008b). Light-Induced Orientational Effects in Periodic Photonic Structures with Pure and Dye-Doped Nematic Liquid Crystal Defects. Physical Review A, Vol. 78, No. 5, pp. 053823

Morris, S. M.; Ford, A. D.; Pivnenko, M. N. \& Coles, H. J. (2005). Enhanced Emission from Liquid-Crystal Lasers. Journal of Applied Physics, Vol. 97, No. 2, pp. 023103

Nelson, B. E.; Gerken, M.; Miller, David A. B.; Piestun, R.; Lin, C.-C. \& Harris, James S. (2000) Use of a Dielectric Stack as a One-Dimensional Photonic Crystal for Wavelength Demultiplexing by Beam Shifting. Optics Express, Vol. 25, No. 20, pp.1502-1504

Ozaki, R.; Matsuhisa, Y.; Ozaki, M. \& Yoshino, K. (2005). Low Driving Voltage Tunable Laser based on One-Dimensional Photonic Crystal Containing Liquid Crystal Defect Layer. Molecular Crystal and Liquid Crystals, Vol.441, No. 1, pp. 441, 87-95

Ozaki, R.; Matsui, T.; Ozaki, M. \& Yoshino, K. (2002). Electro-Tunable Defect Mode in OneDimensional Periodic Structure containing Nematic Liquid Crystal as a Defect Layer. Japanese Journal of Applied Physics, Vol. 41, No. 12B, pp. L1482-L1484

Ozaki, R.; Matsui, T.; Ozaki, M. \& Yoshino, K. (2003a). Electrically Color-Tunable Defect Mode Lasing in One-Dimensional Photonic-Band-gap System containing Liquid Crystal. Applied Physics Letters, Vol. 82, No. 21, pp. 3593-3595

Ozaki, R.; Matsui, T.; Ozaki, M. \& Yoshino, K. (2004a). Optical Property of Electro-Tunable Defect Mode in 1D Periodic Structure with Light Crystal Defect Layer. Electronics and Communications in Japan, Vol. 87, No. 5, pp. 24-31

Ozaki, R.; Moritake, H.; Yoshino, K. \& Ozaki, M. (2007). Analysis of Defect Mode Switching Response in One-Dimensional Photonic Crystal with a Nematic Liquid Crystal Defect Layer. Journal of Applied Physics, Vol. 101, No. 3, pp. 033503

Ozaki, R.; Ozaki, M. \& Yoshino, K. (2003b). Defect Mode Switching in One-Dimensional Photonic Crystal with Nematic Liquid Crystal as Defect Layer. Japanese Journal of Applied Physics, Vol. 42, No. 6B, pp. L669-L671

Ozaki, R.; Ozaki, M. \& Yoshino, K. (2004b). Defect Mode in One-Dimensional Photonic Crystal with In-Plane Switchable Nematic Liquid Crystal Defect Layer. Japanese Journal of Applied Physics, Vol. 43, No. 11B, pp. L1477-L1479

Painter, O.; Lee, R. K.; Scherer, A. Yariv; A. O'Brien, J. D.; Dapkus, P. D. \& Kim, I. (1999). Two-Dimensional Photonic Band-gap Defect Mode Laser. Science, Vol. 284, No. 5421, pp. 1819-1821

Park, B.; Kim, M.; Kim, S. W. \& Kim, I. T. (2009). Circularly Polarized Unidirectional Lasing from a Cholesteric Liquid Crystal Layer on a 1-D Photonic Crystal Substrate. Optics Express, Vol. 17, No. 15, pp. 12323-12331

Park, S. H.; Xia, B. \& Gates, Y. (1999). A Three-Dimensional Photonic Crystal Operating in the Visible Region. Advance Materials, Vol. 11, No. 6, 462-466 
Timofeev, I. V.; Lin, Y.-T.; Gunyakov, V.A.; Myslivets, S. A.; Arkhipkin, V. G.; Vetrov, S. Ya.; Lee, W. \& Zyryanov, V. Ya. (2012). Voltage-Induced Defect Mode Coupling in a OneDimensional Photonic Crystal with a Twisted-Nematic Defect Layer. Physical Review E, Vol. 85, No. 1, pp. 011705

Wen, C.-H. \& Wu, S.-T. (2005). Dielectric Heating Effects of Dual-Frequency Liquid Crystals. Applied Physics Letters, Vol. 86, No. 23, pp. 231104

Wu, S.-T. \& Wu, C.-S. (1996). Mixed-Mode Twisted Nematic Liquid Crystal cells for Reflective Displays. Applied Physics Letters, Vol. 68, No. 11, pp. 1455-1457

Wu, C.-Y.; Zou, Y.-H.; Timofeev, I.; Lin, Y.-T.; Zyryanov, V. Ya.; Hsu, J.-S. \& Lee, W. (2011). Tunable Bi-functional Photonic Device based on One-Dimensional Photonic Crystal infiltrated with a Bistable Liquid-Crystal Layer. Optics Express, Vol. 19, No. 8, pp. 73497355

Xianyua, Haiqing; Wu S.-T. \& Lin, C.-L. (2009). Dual Frequency Liquid Crystals: a Review. "Liquid Crystals, Vol. 36, No. 6-7, pp. 717-726

Xu, M. \& Yang, D.-K. (1997). Dual Frequency Cholesteric Light Shutters. Applied Physics Letters, Vol. 70, No. 6, pp. 720-722

Yablonovitch, E. (1987). Inhibited Spontaneous Emission in Solid-State Physics and Electronics. Physical Review Letters, Vol. 58, No. 20, pp. 2059-2062

Yao, I.-A.; Yang, C.-L.; Chen, C.-J.; Pang, J.-P.; Liao, S.-F.; Li, J.-H. \& Wu, J.-J. (2009). Bistability of Splay and $\pi$-Twist States in a Chiral-Doped Dual Frequency Liquid Crystal Cell. Applied Physics Letters, Vol. 94, No. 7, pp. 071104

Yeh, P. H. \& Gu, C. (1999a). Normal Modes of Propagation in a General TN-LC. In: Optics of Liquid Crystal Display, pp. 130-136, John Wiley \& Sons, ISBN 0-471-18201-X, Canada

Yeh, P. H. \& Gu, C. (1999b). Light Propagation in Uniaxial Media. In: Optics of Liquid Crystal Display, pp. 63-68, John Wiley \& Sons, ISBN 0-471-18201-X, Canada

Yeung, Fion S.-Y.; Xie, F.-C.; Wan, Jones T.-K.; Lee, F. K.; Tsui, Ophelia K. C.; Sheng, P. \& Kwok, H.-S. (2006). Liquid Crystal Pretilt Angle Control using Nanotextured Surfaces," Journal of Applied Physics, Vol. 99, No. 12, pp. 124506

Yin, Y.; Shiyanovskii, S. V. \& Lavrentovich, O. D. (2006). Electric Heating Effects in Nematic Liquid Crystals. Journal of Applied Physics, Vol. 100, No. 2, pp. 024906

Yokoyama, S.; Mashiko, S.; Kikuchi, H.; Uchida, K. \& Nagamura, T. (2006). Laser Emission from a Polymer-Stabilized Liquid-Crystalline Blue Phase. Advance Materials, Vol. 18, No. 1 , pp. 48-51

Yu, H.; Tang, B.; Li, J. \& Li, L. (2005). Electrically Tunable Lasers made from ElectroOptically Active Photonics Band gap Materials. Optics Express, Vol. 13, No. 18, pp. 72437249

Zyryanov, V. Ya.; Gunyakov, V. A.; Myslivets, S. A.; Arkhipkin, V. G. \& Shabano, V. F. (2008). Electrooptical Switching in a One-Dimensional Photonic Crystal. Molecular Crystal and Liquid Crystals, Vol. 488, No. 1, pp. 118-126

Zyryanov, V. Ya.; Myslivets, S. A.; Gunyakov, V. A.; Parshin, A. M.; Arkhipkin, V. G.; Shabanov,V. F. \& Lee, W. (2010). Magnetic-Field Tunable Defect Modes in a PhotonicCrystal/Liquid-Crystal Cell. Optics Express, Vol. 18, No. 2, pp. 1283-1288 